\title{
A propósito de la intervención del castillo de Matrera (Villamartín, Cádiz). Estrategias de intervención de las fortificaciones en España
}

El polémico proyecto de consolidación de la fortaleza de Villamartín, incluido en 2014 en la lista roja de patrimonio elaborada por Hispania Nostra, recibe también premios internacionales, como el Architizer A+ en 2016. La aplicación correcta de la normativa española en materia de conservación del patrimonio exige una comprensión profunda de los principios que la motivaron, coherencia entre la ética profesional y los fines propuestos, y establecer como objetivo prioritario y fundamental la protección y conservación de los bienes culturales. Aplicado al castillo de Matrera, ¿se ha llevado al extremo la legislación sobre restauración?

Irene Merino Mena | conservadora-restauradora de patrimonio

URL de la contribución <www.iaph.es/revistaph/index.php/revistaph/article/view/3777>

Además del desarrollo metacognitivo, adquirido normalmente con la formación y la experiencia, los criterios metodológicos requieren de su adaptación mediante el análisis específico del caso concreto. Aplicado al castillo de Matrera, a grandes rasgos, esto conlleva el estudio de la tipología concreta de la fortificación y de la viabilidad para su uso posterior (acceso, visita, proyectos de musealización, etc).

La conservación, restauración y rehabilitación de un bien como éste, con un nivel de protección BIC y por tanto, inscrito en el Catálogo General del Patrimonio Andaluz, exige también cumplir con la legislación relativa al Proyecto de intervención en la Ley 14/2007 del Patrimonio Histórico de Andalucía ${ }^{1}$.

Esta ley recoge el contenido del proyecto, que puede considerarse como la base argumental necesaria para plantear las intervenciones, ya que atienden tanto al estudio del propio inmueble como a los valores culturales intrínsecos al mismo, la evaluación y diagnóstico de su estado de conservación, una propuesta de conservación desde su perspectiva teórica, técnica y económica, las consecuencias sobre los valores protegidos y por último, un plan que permita su conservación a largo plazo.

A pesar del elevado desconocimiento que existe en general sobre las fortificaciones en España², el casti- llo de Matrera empezó a ser investigado a partir de las descripciones de los restos hallados a principios del siglo $X X^{3}$, se estudió con métodos de análisis estructural y funcional ${ }^{4}$, prospecciones arqueológicas ${ }^{5}$ y parte de las investigaciones más recientes fueron promovidas por el Museo Histórico Municipal de Villamartín en el año 2003 (GUTIÉRREZ LÓPEZ; MARTÍNEZ ENAMORADO, 2003: 104).

Sabemos que esta fortaleza constituyó la vanguardia del cinturón exterior de la "banda morisca" (GUTIÉRREZ LÓPEZ; MARTÍNEZ ENAMORADO, 2003: 103) durante el siglo XIII. Formaba parte de un conjunto de castillos destinados a defender los territorios del Reino de Sevilla de las incursiones nazaríes desde el otro lado de la demarcación, especialmente zonas inmediatas como Zahara, Aznalmara y Cardela.

Además y, como resultado de estos estudios históricos y arqueológicos, el castillo de Matrera ha posibilitado que se plantearan hipótesis referentes a los momentos previos al período bajomedieval, hallándose vestigios propios de época orientalizante e ibérica (GUTIÉRREZ LÓPEZ; MARTÍNEZ ENAMORADO, 2003: 113).

Villamartín, como territorio fronterizo, ha sufrido los avatares propios de una intensa actividad bélica, a los que se suman determinados acontecimientos históri- 
$\cos ^{6}$ que definieron el período bajomedieval en la atalaya situada sobre la campiña del Guadalete. Por tanto, constituye una fuente de conocimiento acerca de la sociedad y su desarrollo, especialmente de la España de la Reconquista y su condición respecto al dominio almohade.

No obstante, la significación del castillo de Matrera comprende también los datos que nos han permitido conocer el proceso evolutivo que tiene como fortificación en sí misma, ejemplo de arquitectura defensiva compuesto por una serie de elementos interrelacionados entre sí y con significado propio: diferentes ladrillos, piedras, cerámicas, argamasas, etc.

A raíz de esto, conviene considerar que las fortificaciones suelen coincidir en una característica constructiva: su complejidad estratigráfica. La superposición de los distintos cuerpos de fábrica para engrosar los muros mediante la sucesión de estratos, generando diferentes etapas constructivas con el tiempo, es común en este tipo de arquitectura en la que, debido a su funcionalidad defensiva, se requería del sucesivo engrosamiento de los muros.

Esto constituye un factor determinante en su estudio y de cara a su intervención, ya que implica que los niveles más antiguos permanecen ocultos en el interior y por tanto, si se basa únicamente en la microestratigrafía de los paramentos resultará poco representativo ${ }^{7}$ porque no tendría en cuenta su naturaleza fragmentaria y diacrónica así como tampoco la identidad múltiple del inmueble, interrelacionando las etapas, los aparejos y el grado de diferenciación entre sí, como identitarios (COBOS GUERRA; RETUERCE VELASCO, 2011: 17).

Por tanto, la estrategia más conveniente de cara a la intervención de las fortificaciones será la que parta de su concepción como un conjunto de estructuras y fábricas pertenecientes a los diferentes momentos históricos con estudios tridimensionales. Todas estas fases constituyen la vida del monumento.

Como consecuencia, considerar únicamente un período en su historia para interpretar esta construcción, conlle- varía no sólo despreciar los demás, sino también el detrimento de su autenticidad y originalidad.

Curiosamente, este caso particular puede servir para ilustrar la diferencia entre dos posturas antagónicas respecto a la interpretación de los criterios de restauración de inmuebles: el repristino historicista frente a la disonancia estética (COBOS GUERRA; RETUERCE VELASCO, 2011: 112-113).

Si aceptamos una tercera postura, la que se basa en una metodología documental, la recuperación de la volumetría con un mortero de cal podría atribuirse a los restos hallados durante el registro arqueológico publicado en el 2003 (GUTIÉRREZ LÓPEZ; MARTÍNEZ ENAMORADO, 2003: 108-111) en la muralla para la cementación de los bloques y el enlucimiento de los muros. También podría deberse al tapial que se encuentra por delante del recinto, cuyo paramento se constituye de cal con zócalo de mampostería. Además, se planteó la hipótesis de que este tapial se tratara de un antemuro de tabiya previo, posibilitando la presencia de una antigua estructura castral de época andalusí.

La elección de un mortero bastardo de cal en esta reintegración con un acabado de color blanco, si se basa como afirma el arquitecto artífice en que la torre "estaba revestida de un mortero, de argamasa blanca" (ARRIZABALAGA, 2016) deja abierto un amplio margen de dudas considerando las limitadas pruebas de la existencia del mismo. Esta estrategia manifestaría que la intervención tuvo la pretensión de devolver al monumento a un estado "original", ya que muestra una predilección por un material atribuido a un estado concreto frente a la diversidad de etapas y aparejos que constituye una fortificación como el castillo de Matrera, como ya corroboraban los resultados de los estudios que preceden a la intervención.

Podemos concluir que quizás la estrategia que habría resultado más acorde a la preservación de los valores intrínsecos de estos monumentos arquitectónicos sea la que considere estos bienes como documentos, permita su lectura completa de los diferentes estratos estratigráficos y deje abierta la posibilidad de que continúen las 


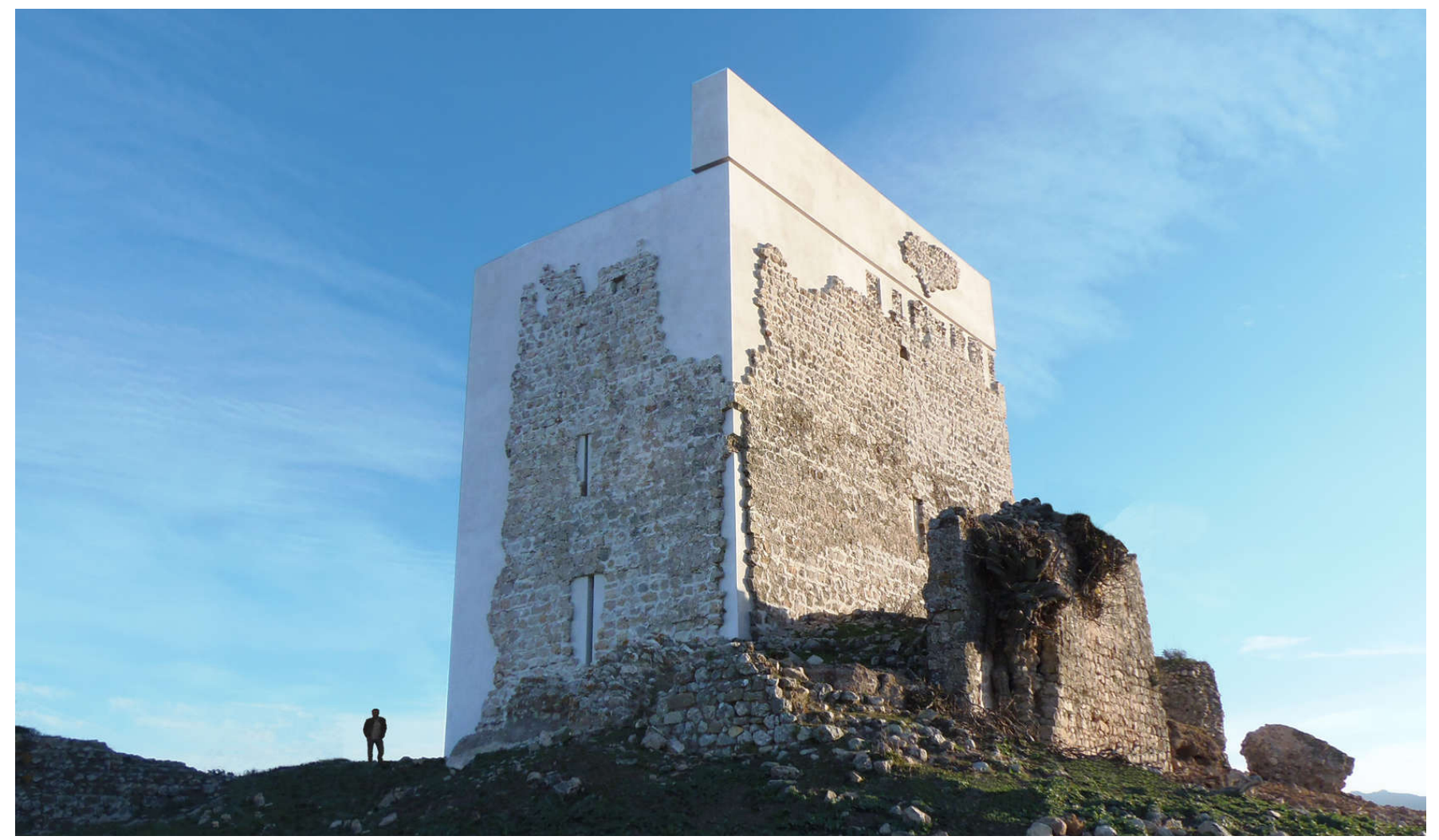

Castillo de Matrera después de su intervención I foto Carquero arquitectura

investigaciones futuras basadas en las evidencias sin resolver, el proceso evolutivo del edificio y la consideración de sus elementos como rasgos documentales integrantes del conjunto con importancia similar.

Cabe exigir que las intervenciones de los bienes culturales, aun siendo realizadas bajo presupuestos particulares debido a su régimen de propiedad e intervenidos por profesionales que no son propiamente conservadores-restauradores, permitan la interpretación de su autenticidad y eviten tanto las falsificaciones como las disonancias estéticas.

La garantía de que la restauración persiga el objetivo prioritario de la conservación del monumento puede partir de la estrategia de intervención, basándose no sólo en un estudio histórico y artístico en profundidad sino además, en posibilitar la apreciación de estos valores considerando que no está orientada únicamente a la comprensión de los especialistas, sino que debe ser extensible al conjunto de los ciudadanos. De otro modo, limitaría e incluso puede llegar a distorsionar completamente su interpretación, al no permitir que se aprecie en su medida las diferentes aportaciones al contemplar el monumento por sí mismos.

Es fundamental tener esto en cuenta ya que la ética profesional puede extenderse a una concienciación pública, reforzando además que la sociedad valore y se implique en la protección de los bienes culturales y en paralelo a esto, también en la educación a través de su difusión.

Al fin y al cabo, fue una sociedad concreta la que originó el castillo de Matrera con un relato propio cuyo desarrollo está patente en todos sus rasgos y, al mismo tiempo, es incuestionable la relación actual con la sociedad a la que pertenece, como además forman parte de la arquitectura defensiva dentro del conjunto del patrimonio histórico de Españå (CARTA, 2006: 1). 


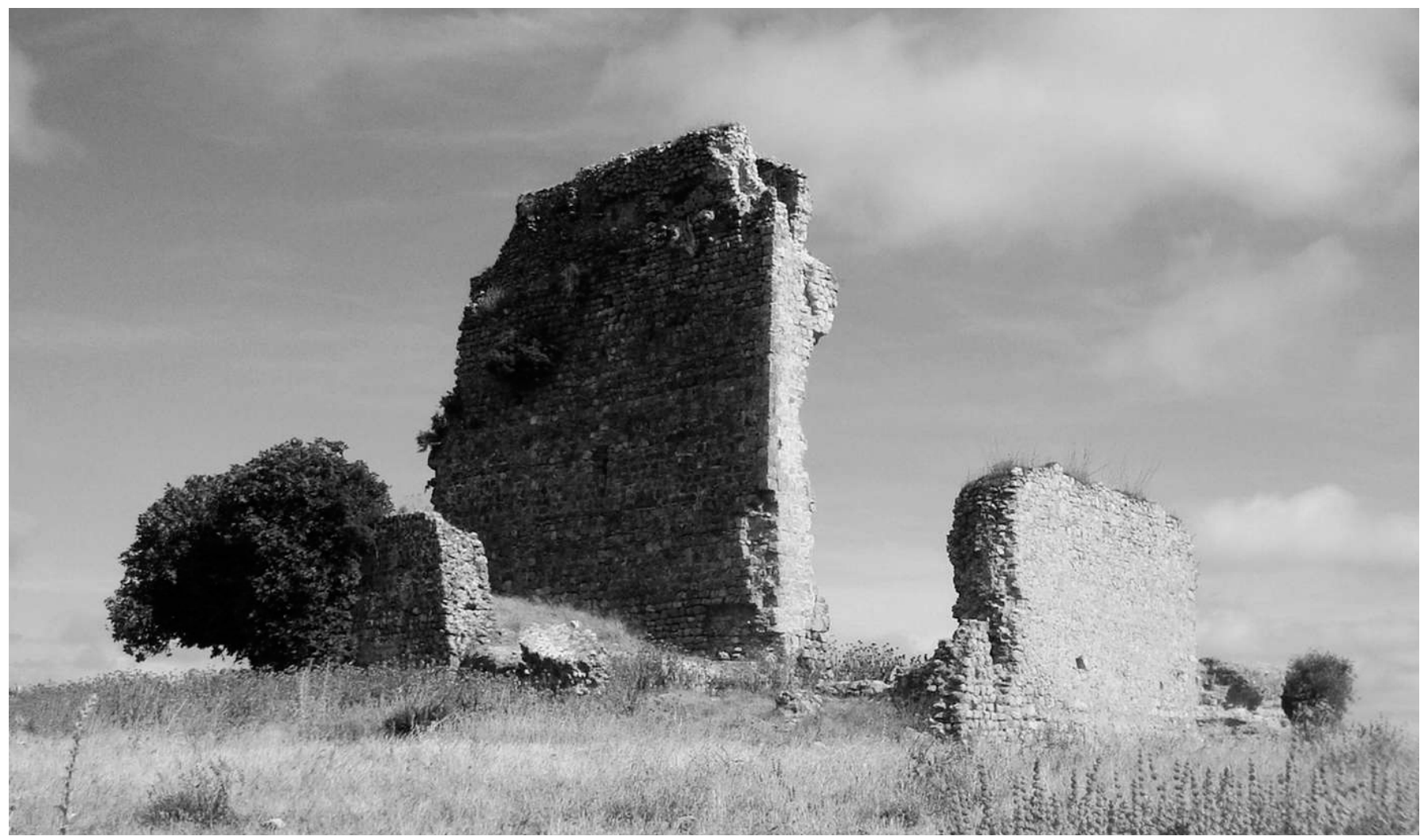

Castillo de Matrera antes de su intervención I foto Carquero arquitectura

Podría entenderse como que, al igual que entre las diferentes etapas constructivas que constituyen su estructura, se establece un vínculo entre los diferentes momentos históricos que en las que se originaron, estableciendo un diálogo, más allá de las limitaciones cronoespaciales, en un mismo momento y lugar.

A través del paso del tiempo y de su correcta interpretación, el monumento arquitectónico puede constituir la llave que permita, a cada nuevo visitante, abrir la puerta hacia su propia memoria heredada.

\section{NOTAS:}

1. El proyecto es un paso previo imprescindible a la restauración, ya que constituye la base sobre la que se articula la intervención, el medio por el que se autoriza la misma y acompañado del informe de ejecución de las intervenciones, la documentación fundamental para que la Administración pueda ejercer su labor preventiva (antes de la intervención, la aprobación del proyecto), la inspección durante la ejecución del mismo y el análisis que permite evaluar el proceso completo después de que terminen los trabajos de intervención previstos.

2. Esto se debe a varios factores: la falta de estudios generales sobre fortificación española, la clasificación de las mismas atendiendo a un orden poco práctico para su consideración como sistemas en los que establecer relaciones y comparativas, la ausencia de rasgos que permitan su adscripción como tipologías arquitectónicas en la Historia del Arte y la necesidad de investigación en fondos de archivo con datos ajenos a aspectos rela- 
cionados con su arquitectura o arqueología, entre otros (COBOS GUERRA; RETUERCE VELASCO, 2011:12-13)

3. Comenzando por Miguel Mancheño y Olivares y Pedro Pérez Clotet, a los que sucedieron otros investigadores que glosaron lo expuesto por los anteriores.

4. «Esquema Gaditano» de finales del siglo XX, del cual tenemos referencia por L. De Mora-Figueroa.

5. Prospección arqueológica de 1999, con el objeto de cartografiar indicios de una edificación andalusí previa.

6. Destacan: la anexión y conquista castellana del campo de Matrera, el sometimiento de la población mudéjar mediante pactos (1225-1250), la incorporación de la fortificación al alfoz de Arcos (1253) al pasar a pertenecer a la Orden de Calatrava, la cesión de Alfonso $X$ de la villa y el castillo (1256), la participación de los mudéjares habitantes tras la gran rebelión en Murcia y en el valle del Guadalete y su posterior expulsión (1264). Más adelante, su constitución como frontera entre territorios divididos por la guerra, la amenaza constante de los meriníes, el asedio y entrega por capitulación de la fortaleza con Alfonso XI con la consecuente cesión del castillo al Concejo de Sevilla, con el compromiso de repoblar y ordenar con la población anexa de Hortales. Finalmente, el castillo de Matrera se constituye como exponente de la conocida "banda morisca" (GUTIÉRREZ LÓPEZ; MARTÍNEZ ENAMORADO, 2003: 103-108).

7. Se limita al estudio de los paramentos y las superficies epiteliales.

8. "El término Arquitectura Defensiva comprende al conjunto de estructuras que se han construido a lo largo de la historia para la defensa y el control de un territorio, del cual forman parte indisoluble. Esta arquitectura constituye una de las expresiones más claras e inteligibles de la historia, ligada de forma muy directa a acontecimientos transcendentes y a personas y colectividades que han jugado papeles relevantes a lo largo de los tiempos. Su presencia en lugares estratégicos, tanto en poblaciones como en el medio natural, ha provocado su integración como elementos singulares en el paisaje natural y urbano".

\section{BIBLIOGRAFÍA}

- ARRizabalagA, M. (2016) Se ha buscado el acabado que tenía la torre del castillo de Matrera en su origen. $A B C, 10$ de marzo de 2016 [en línea] <http://www.abc.es/cultura/abcipolemica-cadiz-buscado-acabado-tenia-torre-castillo-matreraorigen-201603101500_noticia.html> [Consulta: 25/04/2016]

- CONSTITUCIÓN ESPAÑOLA 1978. Boletín Oficial del Estado, n. ${ }^{\circ} 311$, de 29 de diciembre de 1978 (art. 44, 46, 148y 149) <https://www.boe.es/buscar/act.php?id=BOE-A-1978-31229> [Consulta: 25/04/2016]

- CARTA de Baños de la Encina para la conservación de la arquitectura defensiva en España (2006). En Instituto de Patrimonio Cultural de España. Ministerio de Cultura [en línea] <http://ipce.mcu.es/pdfs/BaniosEncina.pdf> [Consulta: 25/04/2016]

- CARTA Internacional para la Conservación y Restauración de Monumentos (Carta de Venecia 1964). II Congreso Internacional de Arquitectos y Técnicos de Monumentos Históricos, Venecia, 1964. En ICOMOS [en línea] <http://www. icomos.org/charters/venice_sp.pdf> [Consulta: 25/04/2016]

- COBOS GUERRA, F.; RETUERCE VELASCO, M. (2011) Metodología, valoración y criterios de intervención en la arquitectura fortificada de Castilla y León: Catálogo de las provincias de León, Salamanca, Valladolid y Zamora. Junta de Castilla y León [en línea] 2011 <http://eprints.ucm.es/23418/1/ Cobos\%26Retuerce_Arquit_fortificada.pdf> Valaldolid: Junta de Castilla y León, 2011 [Consulta: 25/04/2016]

- coBos, F. (2002) Lectura estratigráfica y restauración de fábricas. En Actas de la I Bienal de Restauració Monumental : L'Hospitalet de Llobregat (Barcelona), del 23 al 26 de niviembre del 2000. Barcelona: Dipuació de Barcelona, Servei de Patrimonoi Arquitectónic Local, 2002 <http://www. academiadelpartal.org/contenidos/uploads/actas_i_bienal_03. pdf> [Consulta: 27/04/2016]

- COMPETENCIAS necesarias para acceder a la profesión de conservador-restaurador En ECCO: European Confederation Of Conservator-Restorers' Organisations [en línea] <http:// www.ecco-eu.org/fileadmin/assets/documents/publications/ ECCO_Competencias_ES.pdf> [Consulta: 25/04/2016]

- ESPINOSA, P. (2013) La última bóveda del castillo medieval de Villamartín se derrumba. El País, 16 de abril de 2013 [en línea] <http://ccaa.elpais.com/ccaa/2013/04/16/ andalucia/1366133824_899681.html> [Consulta: 25/04/2016]

- La JUNTA se queja de "intrusiones" militares en el castillo de Matrera de Villamartín, declarado BIC (2013). Europa Press, 3 de septiembre de 2013 [en línea] <http://www. europapress.es/andalucia/cadiz-00351/noticia-junta-quejaintrusiones-militares-castillo-matrera-villamartin-declaradobic-20140903132042.html> [Consulta: 25/04/2016]

- Se derrumba en VILLAMARTín "gran parte" del castillo medieval de Matrera (Cádiz) (2013) En Arqueología medieval 
(Grupo de investigación Toponimia, historia y arqueología del Reino de Granada), 17 de abril de 2013 [en línea] <http:// www.arqueologiamedieval.com/noticias/8420/se-derrumbaen-villamartin-gran-parte-del-castillo-medieval-de-matreracadiz> [Consulta: 25/04/2016] (Fuentes: Armario, E. (2013) Diario de Cádiz 17/04/2013 <http://www.diariodecadiz.es/ article/ocio/1503689/emblema/villamartin/se/derrumba. htmll; Algecirasalminuto.com 16/04/2013 <http://www. algecirasalminuto.com/index.php/noticias/sucesos/47893-sederrumba-en-villamartin-qgran-parteq-del-castillo-medievalde-matrera-catalogado-como-bic>)

- La JUNTA niega dejación en el derrumbe del Castillo de Matrera (Cádiz) (2013) En Arqueología medieval (Grupo de investigación Toponimia, historia y arqueología del Reino de Granada), 12/05/2013 [en línea] <http://www. arqueologiamedieval.com/noticias/8509/la-junta-niegadejacion-en-el-derrumbe-del-castillo-de-matrera-cadiz> [Consulta: 25/04/2016] (Fuente: www.sierradecadiz.com)

- GUTIÉRREZ LÓPEZ, J. M.; MARTÍNEZ ENAMORADO, V. (2003) Matrera (Villamartín): una fortaleza andalusí en el Alfoz de Arcos. En Actas del I Congreso de Historia de Arcos de la Frontera con motivo del 750 Aniversario de la Conquista de la Ciudad por Alfonso X "El Sabio" 1253-2003. Días 20, 21 y 22 de Marzo de 2003. Arcos de la Frontera (Cádiz): Ayuntamiento de Arcos de la Frontera, 2003

- El ALCALDE de Villamartín defiende la restauración del castillo de Matrera tras la polémica. La voz de Cádiz, 11 de marzo de 2016 [en línea] <http://www.lavozdigital.es/cadiz/ sierra/lvdi-alcalde-villamartin-defiende-restauracion-castillomatrera-tras-polemica-201603111855_noticia.html> [Consulta: 25/04/2016]

- LEY 1/1991, de 3 de julio, de Patrimonio Histórico Andaluz. Boletín Oficial del Estado, n. ${ }^{\circ}$ 178, de 26 de julio de 1991, pp. 24826-24836 <http://juntadeandalucia.es/boja/1991/59/1> [Consulta: 25/04/2016]

- LEY de Patrimonio Histórico Español, de 13 de mayo de 1933, modificada por la de 22 de diciembre de 1955 sobre defensa, conservación y acrecentamiento del patrimonio histórico-artístico nacional <http://www.boe.es/datos/pdfs/BOE /1933/145/A01393-01399.pdf> [Consulta: 25/04/2016]

- LEY ORGÁNICA 16/1985, de 25 de junio, del Patrimonio Histórico Español. Boletín Oficial del Estado, n. ${ }^{\circ}$ 155, de 29 de junio de 1985, pp. 20342-20352 <https://www.boe.es/buscar/ doc. php?id=BOE-A-1985-12534> [Consulta: 25/04/2016]

- LISTA Roja del Patrimonio. Ficha Castillo de Matrera <http:// listarojapatrimonio.org/ficha/castillo-de-matrera/> [Consulta: 25/04/2016]

- MACARRóN, A. (2008) Conservación del Patrimonio Cultural. Criterios y normativas. Madrid: Síntesis, 2008
- IZQUIERDA Unida lleva al Parlamento Andaluz la polémica por la restauración del castillo de Matrera. Sierra de Cádiz, 13 de marzo de 2016 <http://www.sierradecadiz.com/2016/03/13/ izquierda-unida-lleva-al-parlamento-andaluz-la-polemica-porla-restauracion-del-castillo-de-matrera.html> [Consulta: 25/04/ 2016] 